\title{
ALGORITHM FOR SHIFTING POSITRON ANNIHILATION SPECTRA
}

\author{
J. DRYZEK \\ Instutite of Nuclear Physics, Radzikowskiego, 31-343 Kraków 152, Poland \\ AND A. POLAK \\ Cracow University of Technology, Institute of Automobiles and Internal Combustion \\ Engines, Warszawska 24, 31-155 Kraków, Poland
}

\begin{abstract}
We present a simple procedure which allows us to shift the positron annihilation spectra, e.g., the Doppler broadening spectrum of the annihilation line. That is especially required when we wish to compare different spectra obtained with a spectrometer with the reduction of background. As an application of the obtained algorithm we present the studies of positron annihilation in carbon material samples.
\end{abstract}

PACS numbers: $78.70 . \mathrm{Bj}$

\section{Description of the algorithm}

The development of more accurate experimental methods in the positron annihilation field induces a more precise data analysis. Nowadays, measurements of the Doppler broadening (DB) of the annihilation line allow us to obtain a spectrum for which the background to the peak ratio reaches the value of $10^{-6}$. The most interesting part of the spectrum is in the region where the detected events correspond to positron annihilation with core electrons. The low probability of such events induces that they are hardly different from the background.

In many cases a useful analysis of the obtained data consists in the direct comparison of the measured DB spectra and calculation of the ratio or difference spectrum. Nevertheless, from experiment we know that each spectrum from series may exhibit a slight shift within a certain range of channels. This is due to a drift of electronic devices. Thus, the direct comparison of the spectra which are changing within six orders of magnitude may lead to wrong conclusions if an exact correction of the shift is not done. In the paper we propose the numerical procedure which allows us to move the spectrum by any fraction of channel. This procedure is dedicated for the DB spectra, but can be applied to other spectra obtained in nuclear physics. 
Let us assume that the spectrum is described by the function $f(E)$, where $E$ is the energy of the annihilation gamma quanta. Nevertheless, in the measurement we are able to measure only the following integral of the function:

$$
F(i)=\int_{E_{i}}^{E_{i+1}} \mathrm{~d} E f(E)
$$

where $i$ is an integer number representing the channel number. In the measurement, $F(i)$ is the number of counts which were detected in the channel $i$; thus $F(i)$ is an integer function, and let us denote $F(i) \equiv N_{i}$. The set $\left\{N_{1}, \ldots, N_{i}, \ldots, N_{\max }\right\}$ of numbers represents the spectrum measured in a typical experiment using a multichannel analyser. From the calibration of the spectrometer we are able to establish the relation between the energy $E$ of the gamma ray and the number of channel $i$, for simplicity it is a linear function: $E_{i}=A i+B$. If we wish to compare directly two spectra, we should ensure that the energy calibration functions are identical. Let us assume that the gain of the amplifier remains constant, it means that the parameter $A$ is constant, but the electronic drift caused that the parameter $B$ is changing from spectrum to spectrum. The value of $B$ parameter can be established in different ways. If we measure the DB spectrum of the annihilation line, one can assume that the maximum of the spectrum corresponds to the energy $511 \mathrm{keV}$. One of the methods is to fit the DB spectrum by a Gaussian function and from the maximum of this function we may find out the position which corresponds to the energy $511 \mathrm{keV}$. Let us assume that one DB spectrum has its maximum at the channel $k$ and the second one at the channel $l+\varepsilon$, where $k$ and $l$ are integers and $0<\varepsilon<1$. If $\varepsilon$ would be equal to zero, we should only shift the second spectrum by $k-l$ channels before doing their direct comparison, which is a simple task. But the case when $\varepsilon>0$ requires another approach. From experiment we have only the set $\left\{N_{1}, \ldots, N_{i}, \ldots, N_{\max }\right\}$ and from the fitting procedure the value of the $\varepsilon$ parameter is known. Let us assume that locally, in the vicinity of the point $E$, $f(E)$ can be approximated by the linear function, thus

$$
N_{i} \cong \int_{i}^{i+1} \mathrm{~d} E\left(a_{1} E+a_{0}\right)=a_{1}\left(i+\frac{1}{2}\right)+a_{0}
$$

In the same way we can calculate the number of counts in the next channel: $N_{i+1}=a_{1}(i+1)+a_{1} / 2+a_{0}$. From these two equations it is easy to obtain the value of the $a_{1}$ and $a_{0}$ parameters: $a_{1}=N_{i+1}-N_{i}$ and $a_{0}=N_{i}(i+3 / 2)-N_{i+1}(i+1 / 2)$. Now if we apply the linear local approximation of the function $f$, there is no problem to obtain the count in the $i$-th channel shifted by $\varepsilon$ :

$$
N_{i+\varepsilon}=\int_{i+\varepsilon}^{i+l+\varepsilon} \mathrm{d} E f(E) \cong \int_{i+\varepsilon}^{i+1+\varepsilon} \mathrm{d} E\left(a_{1} E+a_{0}\right)=\varepsilon N_{i+1}+(1-\varepsilon) N_{i} .
$$

This is the simplest widely used linear relation which is rather a rough approximation if we wish to shift the DB spectrum. The new spectrum now will be represented by the following set $\left\{N_{1+\varepsilon}, \ldots, N_{i+\varepsilon}, \ldots N_{\max -1+\varepsilon}\right\}$.

Let us develop this procedure in the future, assuming that $f(E)$ can be approximated locally by a polynomial: $g(E)=a_{n} E^{n}+a_{n-1} E^{n-1}+\ldots+a_{0}$. Repeating the above procedure we obtain the following relations for the count in the channel $i$ shifted by $\varepsilon$ : 
for $n=2$

$$
N_{i+\varepsilon}=\frac{\varepsilon^{2}}{2}\left(N_{i-1}-2 N_{i}+N_{i+1}\right)+\frac{\varepsilon}{2}\left(N_{i-1}-N_{i+1}\right)+N_{i}
$$

for $n=3$

$$
\begin{aligned}
N_{i+\varepsilon} & =\frac{\varepsilon^{3}}{6}\left(-N_{i-2}+3 N_{i-1}-3 N_{i}+N_{i+1}\right)+\frac{\varepsilon^{2}}{2}\left(N_{i-1}-2 N_{i}+N_{i+1}\right) \\
& +\varepsilon\left(\frac{1}{6} N_{i-2}-N_{i-1}+\frac{1}{2} N_{i}+\frac{1}{3} N_{i+1}\right)+N_{i},
\end{aligned}
$$

for $n=4$

$$
\begin{aligned}
N_{i+\varepsilon} & =\varepsilon^{4}\left(\frac{1}{24} N_{i-2}-\frac{1}{6} N_{i-1}+\frac{1}{4} N_{i}-\frac{1}{6} N_{i+1}+\frac{1}{24} N_{i+2}\right) \\
& +\varepsilon^{3}\left(-\frac{1}{12} N_{i-2}+\frac{1}{6} N_{i-1}-\frac{1}{6} N_{i+1}+\frac{1}{12} N_{i+2}\right) \\
& +\varepsilon^{2}\left(-\frac{1}{24} N_{i-2}+\frac{2}{3} N_{i-1}-\frac{5}{4} N_{i}+\frac{2}{3} N_{i+1}-\frac{1}{24} N_{i+2}\right) \\
& +\varepsilon\left(\frac{1}{12} N_{i-2}-\frac{2}{3} N_{i-1}+\frac{2}{3} N_{i+1}-\frac{1}{12} N_{i+2}\right)+N_{i},
\end{aligned}
$$

and for $n=5$

$$
\begin{aligned}
N_{i+\varepsilon} & =\varepsilon^{5}\left(-\frac{1}{120} N_{i-3}+\frac{1}{24} N_{i-2}-\frac{1}{12} N_{i-1}+\frac{1}{12} N_{i}-\frac{1}{24} N_{i+1}+\frac{1}{120} N_{i+2}\right) \\
& +\varepsilon^{4}\left(\frac{1}{24} N_{i-2}-\frac{1}{6} N_{i-1}+\frac{1}{4} N_{i}-\frac{1}{6} N_{i+1}+\frac{1}{24} N_{i+2}\right) \\
& +\varepsilon^{3}\left(\frac{1}{24} N_{i-3}-\frac{7}{24} N_{i-2}+\frac{7}{12} N_{i-1}-\frac{5}{12} N_{i}+\frac{1}{24} N_{i+1}+\frac{1}{24} N_{i+2}\right) \\
& +\varepsilon^{2}\left(-\frac{1}{24} N_{i-2}+\frac{2}{3} N_{i-1}-\frac{5}{4} N_{i}+\frac{2}{3} N_{i+1}-\frac{1}{24} N_{i+2}\right) \\
& +\varepsilon\left(-\frac{1}{30} N_{i-3}+\frac{1}{4} N_{i-2}-N_{i-1}+\frac{1}{3} N_{i}+\frac{1}{2} N_{i+1}-\frac{1}{20} N_{i+2}\right)+N_{i} .
\end{aligned}
$$

Note that to perform the shift, we need to know only the whole spectrum and the channel fraction, by which we intend to shift the spectrum. The degree $n$ of polynomial may be chosen depending on the accuracy of subsequent considerations.

To show how the above relations are working, let us generate two spectra which are described by the Gaussian function: $f(E)=N_{\mathrm{g}} \exp \left[-\left(E-E_{0}\right)^{2} / 2 \sigma^{2}\right]$, where $E_{0}=200$ for the reference spectrum and $E_{0}=200.5$ for the second one, $\sigma=18$ and $N_{\mathrm{g}}=10^{6}$ for both spectra. The spectra are presented in Fig. 1a. In Fig. 1b the difference between these two spectra is drawn when no shifting procedure was used. Let us define the coefficient $k^{(n)}$ which says how good is the shifting procedure of order $n: k^{(n)}=N_{\mathrm{d}}^{(n)} / N_{\mathrm{g}}$, where $N_{\mathrm{d}}$ is the maximum difference 


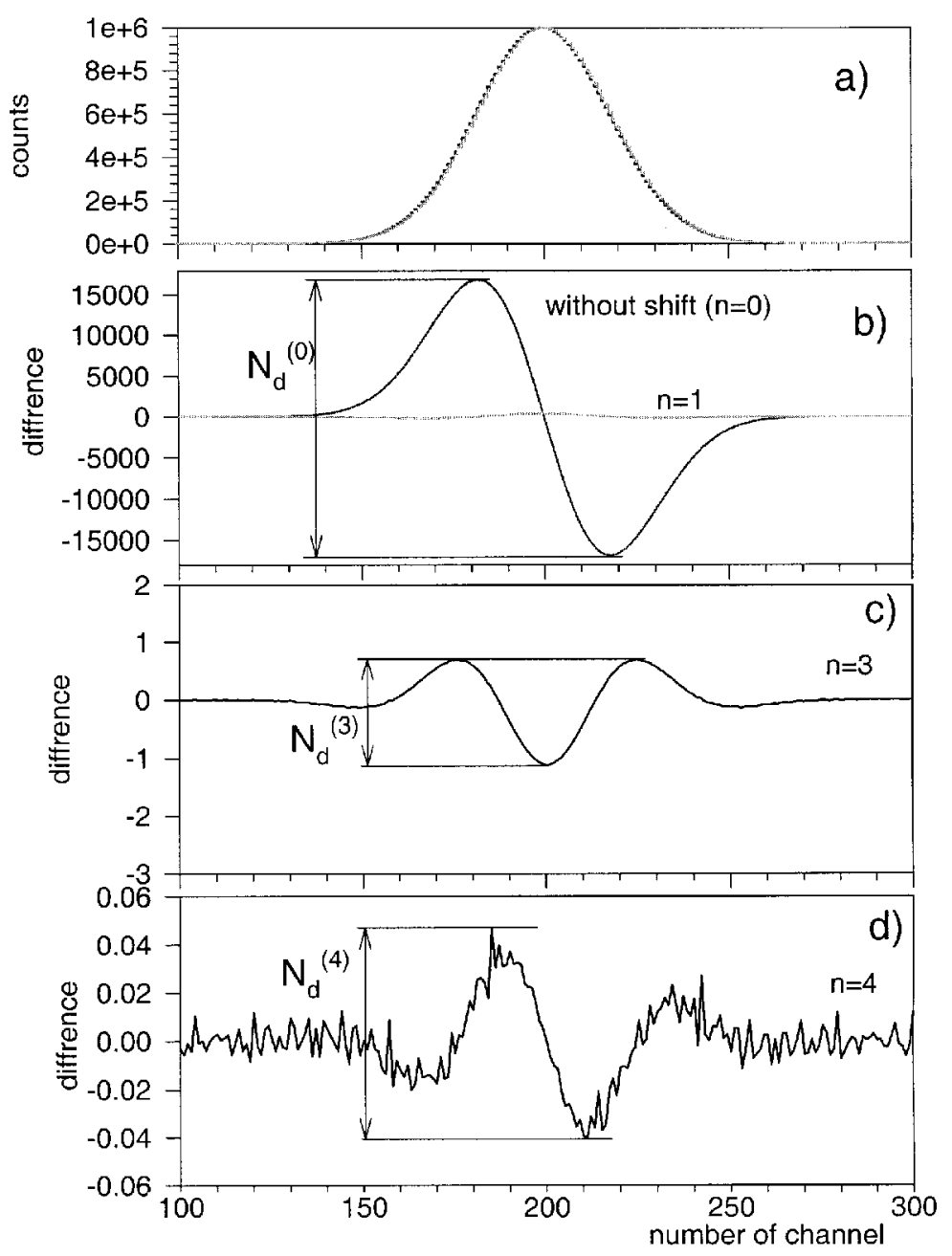

Fig. 1. The two simulated Gaussian spectra, one being shifted with respect to another one by a half of the channel (a). The difference between these two spectra when no shifting procedure was applied and when one spectrum was shifted according to the linear procedure $(n=1)$ (b), and the other procedures with $n=3$ (c) and $n=4$ (d).

between the reference spectrum and the spectrum shifted back by 0.5 channel (see Fig. 1b). For the non shifted spectrum $k^{(0)}=3.4 \times 10^{-2}$, but for $n=1$, $k^{(1)}=3.4 \times 10^{-4}$ from Fig. 1b, $k^{(3)}=1.4 \times 10^{-6}$ from Fig. $1 \mathrm{c}$ and $k^{(4)}=7.4 \times 10^{-8}$ from Fig. 1d. This shows that if we wish to preserve details of the spectrum during the shift procedure, $n$ should by as high as possible. Nevertheless, the presented procedures are not able to shift spectra perfectly and small differences always remain.

Measured spectra always contain a background or statistical noise. Let us find how the character of the noise is changing when the spectrum is shifted with 


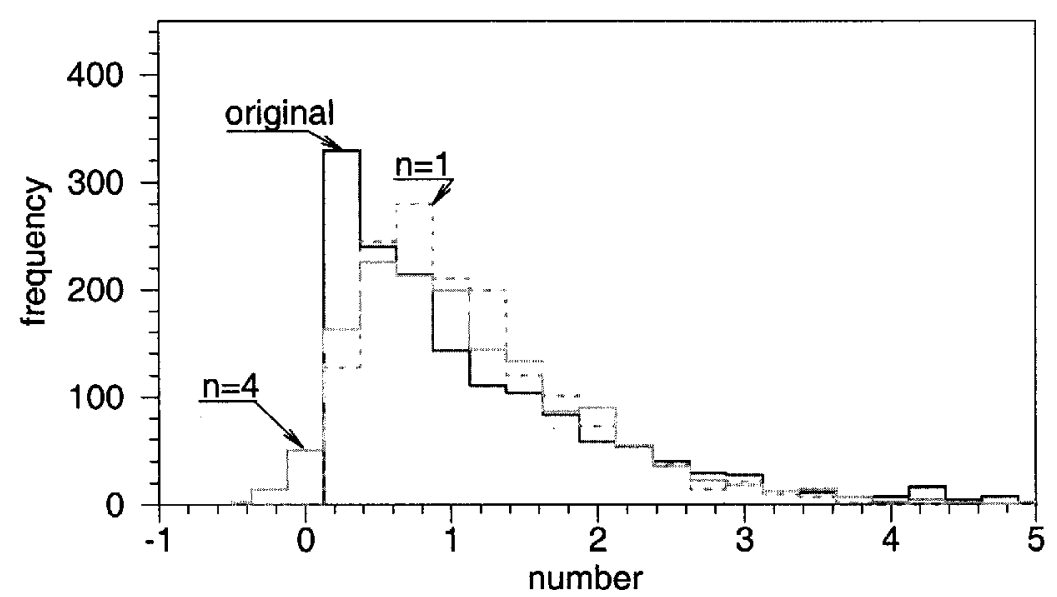

Fig. 2. The distribution of the numbers in the range from 0 to 5 located in 4000 channels. The Poisson distributed numbers represent a noise spectrum. The solid line presents the distribution in the origin noise spectrum, the dotted line - the distribution in the noise spectrum which occurred after shifting the origin spectrum with the linear procedure with $n=1$ and the dashed line with $n=4$.

the above procedure. In 4000 channels we generated the spectrum which contained only the statistical noise. In the channels the random numbers which exhibited the Poisson distribution have been inserted. Note that far away from the annihilation line the DB spectrum is flat and it is similar to our simulated spectrum. The frequency analysis of the spectrum is presented in Fig. 2. Then the spectrum was shifted by $\varepsilon=0.5$ channel using the procedure with $n=1$ and $n=4$ and once again the frequency analysis was performed. The results presented in Fig. 2 show that the shifting procedure changed the shape of the distribution of the statistical noise. Thus, the statistical analysis of the noise should be preferably done on the original spectrum rather than on the shifted one. We believe that this is only a small disadvantage of the presented procedure.

\section{Experimental application of the algorithms}

Let us apply the above presented procedure to experimental data. In our studies we measured the carbon material (CM) samples using the DB spectrometer described in [1]. We wanted to find out whether the presence of pores in the material is reflected in the high momentum part of the DB spectrum. The volume fraction of the graphite in the CM samples produced by the firm Angraph (Poland) was close to $37 \%$. In our former studies of the carbon fibres we have detected two lifetime components in the positron lifetime spectra. The second one $\left(\tau_{2}\right)$ ranged from $373 \mathrm{ps}$ to $441 \mathrm{ps}[2]$ and it can be associated with positron annihilation trapped at pores. The increase in the $\tau_{2}$ value was correlated with the increase in the $S$-parameter defined as the ratio of the central part of the annihilation line to its total area. The measured DB spectrum for each sample exhibited 
a small shift which was evaluated by fitting the Gaussian function. Using the procedure with $n=4$ we have shifted each spectrum to place the maximum of each spectrum to the same integer channel. (After the shift the position of the maximum was checked once again.) For each spectrum the background subtraction procedure was performed as described in Ref. [3]. In Fig. 3a we present the DB spectra measured for the CM samples denoted as E13, E28, and E30, and the $\mathrm{Mg}$ spectrum [4] for comparison. In Fig. 3b we drew the difference between the $\mathrm{Mg}$ and the sample spectrum. Now one can detect only small changes in the region close to $511 \mathrm{keV}$, which corresponds to positron annihilation with electrons with a small momentum. In the region above $515 \mathrm{keV}$, where positron annihilation with core electrons dominates, we did not detect significant differences. In Fig. 4 we show the dependence of the $S$-parameter deduced from Fig. 3a on the longest positron lifetime component detected in the CM samples. (The shortest lifetime component was close to 120 ps.) As we expected, the increase in the positron lifetime caused the increase in the $S$-parameter. We can conclude that the increase in the positron lifetime and the $S$-parameter is related with the positron annihilation in larger pores, which is supported by the fact that we did not see any significant changes in positron annihilation with core electrons. This can be explained that

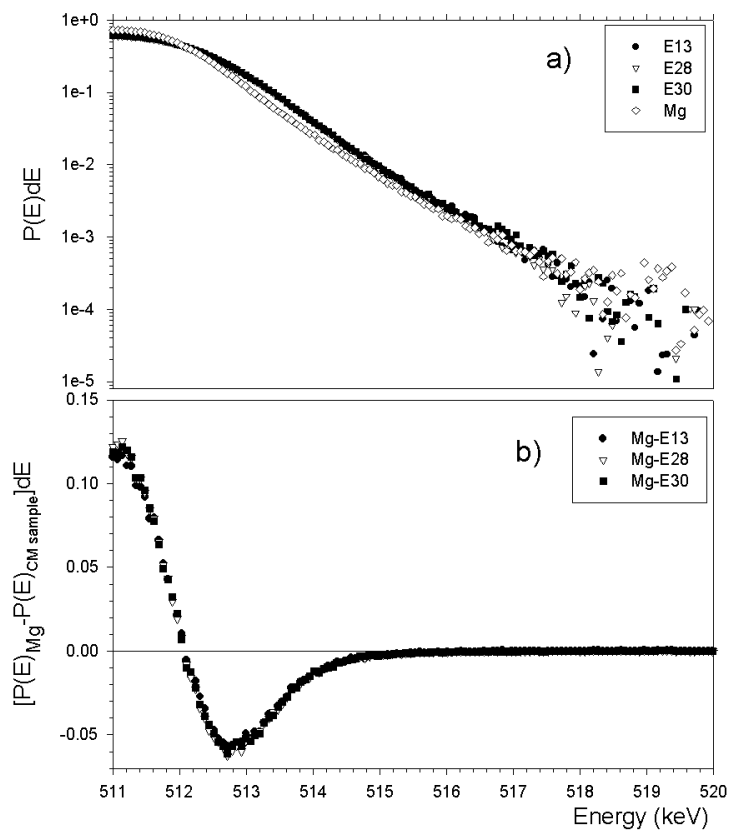

Fig. 3. The Doppler broadening spectra obtained for the CM samples (E13, E28, and E30) and $\mathrm{Mg}$ well-annealed sample (a) and the differences between the $\mathrm{Mg}$ spectrum and spectra of the samples (b). 


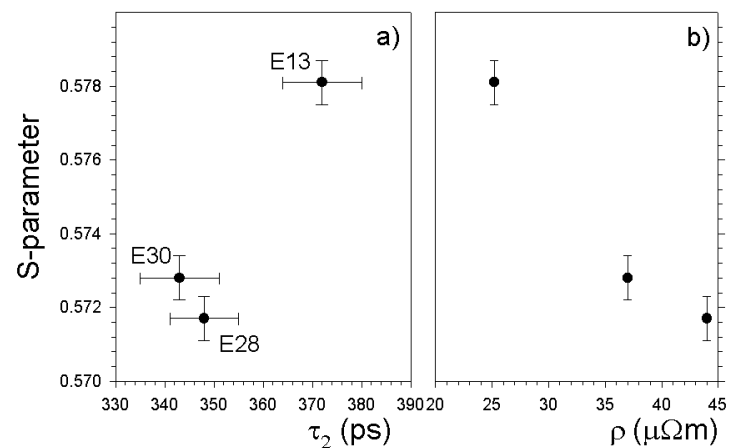

Fig. 4. The value of the $S$-parameter deduced from Fig. 3a vs. the second component of the positron lifetime spectrum (a) and the electrical resistivity (b) measured for the CM samples.

the overlapping of the positron wave function with the core region of the atoms which surrounds the pores is small. It can be happened for a relatively large radius of the pores.

It is interesting to notice that there is a correlation between the $S$-parameter and the electrical resistance of the sample (Fig. 4b). This suggests an influence of the pores on the electrical resistivity of the CM samples.

In conclusion, we presented the procedure for shifting the DB spectra of the annihilation line. Such a shift is sometimes needed for a direct comparison of different spectra. The test of the presented algorithm on the simulated Gaussian spectra showed that the algorithm is quite efficient. Nevertheless, during the shift we may lose the information about the distribution of the noise which is present in the experimental spectra. The algorithm was applied to the study of the DB spectra of the annihilation line for CM samples. From this we could conclude that the presence of pores did not significantly affect the positron annihilation with core electrons in the CM.

The author (A.P.) would like to thank the Committee for Scientific Research (Poland) for supporting this work under the grant No. 9 T12C 04017.

\section{References}

[1] J. Dryzek, E. Dryzek, Nucleonika 42, 87 (1997).

[2] J. Dryzek, E. Pamuła, S. Błażewicz, Phys. Status Solidi A 151, 39 (1995).

[3] J. Dryzek, C.A. Quarles, Nucl. Instrum. Methods Phys. Res. A 378, 337 (1996).

[4] E. Dryzek, J. Kuriplach, J. Dryzek, J. Phys., Condens. Matter 10, 6573 (1998). 